\title{
Flavour models with three Higgs generations
}

\author{
Florian Hartmann ${ }^{\mathrm{a}}$, Wolfgang Kilian \\ Department Physik, Univertity Siegen, 57068 Siegen, Germany
}

Received: 15 May 2014 / Accepted: 31 August 2014 / Published online: 16 September 2014

(C) The Author(s) 2014. This article is published with open access at Springerlink.com

\begin{abstract}
We construct models with a spontaneously broken $\mathrm{SU}(3)_{\mathrm{F}}$ flavour symmetry where three generations of Higgs multiplets transform in a flavour-triplet representation. The models are embedded in a supersymmetric Pati-Salam GUT framework, which includes left-right symmetry. We study the possible flavon representations and show that a model with flavons in the decuplet representation is able to reproduce the hierarchy structure of the quark and lepton mass and mixing matrices. This result requires no additional structure or fine-tunings except for an extra $Z_{4}$ discrete symmetry in the superpotential.
\end{abstract}

\section{Introduction}

The standard model (SM) contains a single complex doublet of scalar fields with one physical Higgs particle, which stands out as an extra field, unrelated to the fermionic matter or gauge fields. The recent discovery at the CERN Large Hadron Collider (LHC) is a clear indication that Higgs bosons exist $[1,2]$. However, with the current data sample, it is not possible to distinguish the minimal Higgs representation from a larger one, in particular from the MSSM Higgs sector or from a model that contains more than one Higgs (bi-)doublet.

In this work, we study the particular case that there are three Higgs generations [3-5] which can be associated with a flavour symmetry [6-8], thus relating the Higgs and matter sectors. Such a structure is present, for instance, in supersymmetric grand-unification models where Higgs and matter fields combine in a common gauge-group representation $[9,10]$.

Extra Higgs generations contribute to the running of the electroweak gauge couplings and therefore adjust the conventional picture of gauge unification. In Ref. [11], we have investigated Pati-Salam models $[13,14]$ and found a considerable number of models with interesting patterns of gauge

a e-mail: hartmann@physik.uni-siegen.de unification that contain three Higgs generations. Similar results can be found in Refs. [15-18].

A flavour symmetry may be either discrete or continuous (for reviews see [19-22]). In this paper, we focus on a continuous $\mathrm{SU}(3)_{\mathrm{F}}$ flavour symmetry. There is a large number of models that realise this idea [22-28], where the $\mathrm{SU}(3)_{\mathrm{F}}$ transformations that apply to left- and right-handed, up- and downtype quarks and leptons, respectively, may be either connected or independent. In a three-generation Higgs model, the Higgs doublets (bi-doublets in the supersymmetric case) also provide a fundamental $\mathrm{SU}(3)_{\mathrm{F}}$ triplet. We consider the simplest possibility that a single $\mathrm{SU}(3)_{\mathrm{F}}$ symmetry universally applies to all Higgs and matter multiplets, and assume that it is spontaneously broken by VEVs of additional scalar fields which we collectively denote as flavons. In order to avoid Goldstone bosons, we assume the flavour SU(3)F to be gauged. Unlike the Higgs scalars, we do not imply that flavons appear at collider energies. ${ }^{1}$

Without further assumptions, one should consider flavons in any representation of $\mathrm{SU}(3)_{\mathrm{F}}$, not just the fundamental triplet representation. As we will show, models with only triplet flavons are feasible, but a model with a dominant decuplet contribution appears more natural and is able to qualitatively accommodate the observed mass- and mixinghierarchy patterns in both the quark and the lepton sectors.

\section{Model framework}

We study extensions of the minimal supersymmetric Standard Model (MSSM) with a gauged SU(3) F flavour symmetry. The flavour symmetry is spontaneously broken, at high energy, by the VEVs of flavon fields $\phi$. Both the three generations of matter and the three generations of Higgs

\footnotetext{
1 We are not concerned with the dynamics of flavon fields, but merely treat them as spurions that parametrise the possible patterns of flavoursymmetry breaking.
} 
bi-doublets transform in the fundamental triplet representation of $\mathrm{SU}(3)_{\mathrm{F}}$. At high energies, we impose Pati-Salam symmetry (PS) which contains a discrete left-right symmetry. This setup allows for complete unification of the SM gauge couplings at or near the Planck scale with a hierarchy of intermediate mass scales [11].

The complete gauge symmetry we start with is thus,

$$
\mathrm{SU}(4)_{\mathrm{C}} \times \mathrm{SU}(2)_{\mathrm{L}} \times \mathrm{SU}(2)_{\mathrm{R}} \times Z_{\mathrm{LR}} \times \mathrm{SU}(3)_{\mathrm{F}} .
$$

We have to treat this as an effective theory expanded in a large (but unspecified) mass scale $M$. In our framework, the flavons are singlets under the PS gauge group and thus do not contribute to the corresponding gauge-coupling scale dependence. As the theory is constructed at a high scale, we also have to take into account all non-renormalizable terms which are not forbidden by a remaining symmetry or by explicit constraints on the low-energy effective theory after symmetry breaking. This allows, in general, mass terms for those components of the flavon superfields that are not absorbed as Goldstone bosons into massive flavour-gauge bosons.

Generically, we denote the quotient of a flavon $\operatorname{VEV}\langle\phi\rangle$ and the expansion scale $M$ by $\epsilon$,

$\epsilon=\frac{\langle\phi\rangle}{M}$.

We assume that $\epsilon$ distinguishes the up- from the down sector, $\epsilon_{d} \neq \epsilon_{u}$, and also allow for a third distinct expansion parameter $\epsilon_{v}$ in the neutrino sector. Such differences are expected, in the low-energy effective superpotential, as a consequence of the breaking of the Pati-Salam gauge symmetry.

An effective Yukawa-coupling matrix with the entry pattern

$Y_{u / d} \approx\left(\begin{array}{ccc}0 & O\left(\epsilon^{3}\right) & O\left(\epsilon^{3}\right) \\ O\left(\epsilon^{3}\right) & O\left(\epsilon^{2}\right) & O\left(\epsilon^{2}\right) \\ O\left(\epsilon^{3}\right) & O\left(\epsilon^{2}\right) & O(1)\end{array}\right) y_{t / b}$,

with $\epsilon_{u} \approx 0.05$ and $\epsilon_{d} \approx 0.15$ can accommodate the observed CKM matrix and the quark-mass hierarchies [29].

In a PS model, the matter spectrum necessary contains right-handed neutrinos. A Majorana mass term for those induces the type-I see-saw mechanism [30-32]. After integrating out the heavy right-handed neutrinos, the effective mass matrix for the SM neutrinos takes the form

$m_{\mathrm{eff}}=m_{\mathrm{LR}} M_{\mathrm{RR}}^{-1} m_{\mathrm{LR}}^{T}$

where $m_{\mathrm{LR}}=Y_{\nu} v_{\mathrm{SM}}$ is the left-right mixing mass matrix from the lepton-Yukawa terms and $M_{\mathrm{RR}}$ is the Majorana mass matrix of the right-handed neutrinos. For the Yukawa structure given above, sequential right-handed neutrino dominance (SRHND) [33] implements a phenomenologically
Table 1 Transformation of the Higgs, matter and flavon superfields responsible for the flavour structure under the gauge and flavour symmetries in the flavon triplet model

\begin{tabular}{lllrrr}
\hline Field & SU(3) F & PS & $U(1)$ & $Z_{3}$ & $Z_{2}^{\mathrm{R}}$ \\
\hline$\Psi_{\mathrm{L}}$ & 3 & $(4,2,1)$ & 1 & 0 & - \\
$\Psi_{\mathrm{R}}$ & 3 & $(\overline{4}, 1,2)$ & 1 & 0 & - \\
$h$ & 3 & $(1,2,2)$ & 1 & 0 & + \\
$\phi_{3}$ & 3 & $(1,1,1)$ & -3 & -1 & + \\
$\overline{\phi_{3}}$ & $\overline{3}$ & $(1,1,1)$ & -1 & 0 & + \\
$\phi_{23}$ & 3 & $(1,1,1)$ & 0 & 1 & + \\
$\phi_{23}$ & $\overline{3}$ & $(1,1,1)$ & -1 & 1 & + \\
$\Phi_{\mathrm{R}}$ & 1 & $(4,1,2)$ & 6 & 0 & + \\
\hline
\end{tabular}

viable PMNS mixing matrix and mass hierarchy in the neutrino sector [22]. Here, a single right-handed neutrino gives the main contribution to the 23 block. A second right-handed neutrino is required to give subdominant contributions to generate the full effective neutrino-mass matrix.

\section{A triplet-flavon model}

We first consider the case that all flavons $\phi$ are in the (anti-) fundamental triplet representation of the flavour symmetry, analogous to matter multiplets $\Psi$ [23-25]. The three generations of Higgs bi-doublets $h$ also transform as a flavour triplet. In order to generate Majorana mass terms for the right-handed neutrinos, we augment this spectrum by a righthanded PS-breaking multiplet $\Phi_{\mathrm{R}}$, as introduced in [11]. We list the fields and quantum numbers that are relevant for the present discussion in Table 1.

The spectrum is completed by further superfields, restoring left-right symmetry as well as cancelling the $\mathrm{SU}(4)_{\mathrm{C}}$ gauge anomaly, and implement symmetry breaking down to the MSSM [11]. Since those superfields do not affect the flavour structure, we do not list them explicitly.

In this model, the Pati-Salam and flavour symmetries allow for the SM gauge couplings and Yukawa terms (see below), but also for extra flavour-dependent interactions which are inconsistent with the observed low-energy flavour phenomenology. We supplement the gauge structure by a $U(1) \times Z_{3}$ symmetry and assign specific charges to the fields, such that unwanted terms are forbidden. (To avoid an extra Goldstone boson, the $U(1)$ may also be considered to be gauged, or we may reduce it to a discrete $Z_{\mathrm{N}}$ sub-symmetry.) These quantum numbers are also given in Table 1. Furthermore, we impose a $Z_{2}^{\mathrm{R}}$ symmetry similar to matter parity.

The particle content of Table 1, including all PS breaking fields, exhibits a $\mathrm{SU}(3)_{\mathrm{F}}$ gauge anomaly. This anomaly can be cancelled by additional fields that do not contribute to the matter flavour structure. For instance, these can be the 
fields $\bar{\phi}_{10}$ and $\phi_{6}$, which transform as $\overline{\mathbf{1 0}}$ and $\mathbf{6}$, respectively, and are PS-singlets. We expect such or similar fields to be introduced in the context of vacuum alignment of the flavon VEVs [12].

We label the flavons $\phi_{i}$ by the directions in flavour space in which their VEVs are aligned. The flavour symmetry is broken in two steps. First we break $\mathrm{SU}(3)_{\mathrm{F}}$ down to $\mathrm{SU}(3)_{\mathrm{F}}$ by the VEV of the flavon field $\phi_{3}$. The field $\phi_{23}$ then further breaks the remaining flavour symmetry. For the sake of simplicity we align the "MSSM-Higgs" VEV along the third component in flavour space. Collecting all flavour-breaking Higgs fields, we propose the vacuum structure

$$
\begin{aligned}
& \left\langle\phi_{3}\right\rangle \sim\left\langle\bar{\phi}_{3}\right\rangle \sim M(0,0, \sqrt[3]{\epsilon}), \\
& \left\langle\phi_{23}\right\rangle \sim\left\langle\bar{\phi}_{23}\right\rangle \sim M(0, \epsilon, \epsilon), \\
& \left\langle\Phi_{\mathrm{R}}\right\rangle \sim M_{\nu}, \\
& \langle h\rangle=(0,0,1) v_{\text {MSSM }} .
\end{aligned}
$$

\subsection{Quark mixing: operator analysis}

We now construct the possible interactions of matter with Higgs and flavon fields that yield contributions to low-energy flavour physics. The effective operators are invariant under Pati-Salam, $\mathrm{SU}(3)_{\mathrm{F}}$, and the extra $U(1)$ and $Z_{3}$ symmetries, but not limited by the condition of renormalizability.

Starting with only the gauge and flavour symmetry of (1), we identify a single dimension-three (renormalizable) term in the superpotential, ${ }^{2}$

$Y_{0}=\varepsilon^{i j k} \Psi_{L, i} \Psi_{R, j} h_{k}$,

where $\varepsilon_{i j k}$ is the three-dimensional Levi-Civita tensor. This term generates a coupling that is non-diagonal among the lepton and quark generations. Taking a look at (3), such a term cannot be the dominant contribution to flavour interactions. It has to be suppressed at least by a factor $\epsilon^{3}$ with respect to the top Yukawa coupling. We choose to eliminate this term by the extra $U(1)$ symmetry mentioned above.

We now consider further operators of increasing dimension, one by one. At dimension four, the algebra of $\mathrm{SU}(3)_{\mathrm{F}}$ does not provide an invariant polynomial that can generate a Yukawa operator.

At dimension five, we again have to forbid all interactions, using the extra symmetries. These operators generate terms of the form

$$
\frac{\bar{\phi} \phi}{M^{2}} Y_{0}
$$

\footnotetext{
${ }^{2}$ We count the dimension in terms of the superpotential, where a dimension-three term is renormalizable, and terms with mass dimension of four and higher are non-renormalizable.
}

which, after symmetry breaking, would re-introduce the universal Yukawa term mentioned above. Its parametric suppression is only of order $\epsilon^{2}$, which is not sufficient.

At dimension six, we finally identify an operator that should contribute to the Yukawa matrices, ${ }^{3}$

$W_{\text {lead }}=\frac{1}{M^{3}}\left(h \bar{\phi}_{3}\right)\left(\Psi_{\mathrm{L}} \bar{\phi}_{3}\right)\left(\Psi_{\mathrm{R}} \bar{\phi}_{3}\right)$.

This term generates Yukawa couplings for the third generation. At the same order, the model is able to seed the 23 block of the Yukawa matrix. The corresponding invariant operator is

$W_{(2,3)}=\frac{1}{M^{3}}\left(h \bar{\phi}_{23}\right)\left(\Psi_{\mathrm{L}} \bar{\phi}_{23}\right)\left(\Psi_{\mathrm{R}} \bar{\phi}_{23}\right)$

It is important that, at this order, the universal term (6) is not generated. As long as there are only two distinct flavon fields involved, this term vanishes by antisymmetry.

In line with the hierarchy pattern in the quark and charged lepton sector, couplings to the first generation and mixing terms are generated by operators of even higher order. Operators of dimension seven and eight do not contribute to the Yukawa matrix in our setup. All sub-leading operators are at least of dimension nine. The corresponding superpotential is

$$
\begin{aligned}
W_{\text {sub }}= & \frac{1}{M^{6}}\left[\left(\phi_{23} \bar{\phi}_{23}\right)^{2}\left(h \bar{\phi}_{23}\right)\left(\phi_{23} \Psi_{\mathrm{L}} \Psi_{\mathrm{R}}\right)\right. \\
& +\left(\phi_{23} \bar{\phi}_{3}\right)^{2}\left(h \bar{\phi}_{3}\right)\left(\phi_{23} \Psi_{\mathrm{L}} \Psi_{\mathrm{R}}\right) \\
& +\left(\phi_{23} \bar{\phi}_{23}\right)^{3}\left(h \Psi_{\mathrm{L}} \Psi_{\mathrm{R}}\right) \\
& +\left(\phi_{23} \bar{\phi}_{3}\right)^{3}\left(h \Psi_{\mathrm{L}} \Psi_{\mathrm{R}}\right) \\
& +\left(\phi_{23} \bar{\phi}_{3}\right)^{2}\left(\Psi_{\mathrm{L}} \bar{\phi}_{3}\right)\left(h \phi_{23} \Psi_{\mathrm{R}}\right) \\
& +\left(\phi_{23} \bar{\phi}_{23}\right)^{2}\left(\Psi_{\mathrm{L}} \bar{\phi}_{23}\right)\left(h \phi_{23} \Psi_{\mathrm{R}}\right) \\
& +\left(\phi_{23} \bar{\phi}_{23}\right)^{2}\left(\Psi_{\mathrm{R}} \bar{\phi}_{23}\right)\left(h \phi_{23} \Psi_{\mathrm{L}}\right) \\
& \left.+\left(\phi_{23} \bar{\phi}_{3}\right)^{2}\left(\Psi_{\mathrm{R}} \bar{\phi}_{3}\right)\left(h \phi_{23} \Psi_{\mathrm{L}}\right)\right] .
\end{aligned}
$$

In summary, we obtain the following parametrical structure of the low-energy effective Yukawa matrices at leading order in $\epsilon$, neglecting all factors of order unity:

$Y_{u / v} \approx\left(\begin{array}{ccc}0 & \epsilon_{u}^{3} & \epsilon_{u}^{3} \\ \epsilon_{u}^{3} & \epsilon_{u}^{2} & \epsilon_{u}^{2} \\ \epsilon_{u}^{3} & \epsilon_{u}^{2} & 1\end{array}\right) \epsilon_{u}, Y_{d / l} \approx\left(\begin{array}{ccc}0 & \epsilon_{d}^{3} & \epsilon_{d}^{3} \\ \epsilon_{d}^{3} & \epsilon_{d}^{2} & \epsilon_{d}^{2} \\ \epsilon_{d}^{3} & \epsilon_{d}^{2} & 1\end{array}\right) \epsilon_{d}$.

\footnotetext{
3 The notation is thus, that a pair of fields should be contracted with a $\delta_{i}^{j}$ and likewise a triple with $\varepsilon_{i j k}$.
} 


\subsection{Neutrino masses and lepton mixing}

We now turn to the neutrino sector. The interplay between the Yukawa and Majorana mass matrices produces a nonhierarchical pattern in the PMNS neutrino mixing matrix, along with the CKM quark mixing matrix as discussed above.

Assuming the quantum numbers given in Table 1, we write down operators that generate a right-handed neutrino Majorana mass, up to dimension 12 :

$$
\begin{aligned}
W_{\text {Maj }}= & \frac{1}{M^{9}} \Phi_{\mathrm{R}}^{2}\left[\left(\phi_{3} \bar{\phi}_{23}\right)^{3}\left(\Psi_{\mathrm{R}} \bar{\phi}_{3}\right)^{2}\right. \\
& +\left(\phi_{3} \bar{\phi}_{23}\right)\left(\phi_{3} \bar{\phi}_{3}\right)^{2}\left(\Psi_{\mathrm{R}} \bar{\phi}_{23}\right)^{2} \\
& +\left(\phi_{3} \bar{\phi}_{3}\right)^{3}\left(\Psi_{\mathrm{R}} \bar{\phi}_{3}\right)^{2} \\
& +\left(\phi_{3} \bar{\phi}_{3}\right)\left(\phi_{3} \bar{\phi}_{23}\right)^{2}\left(\Psi_{\mathrm{R}} \bar{\phi}_{3}\right)\left(\Psi_{\mathrm{R}} \bar{\phi}_{23}\right) \\
& \left.+\left(\phi_{3} \bar{\phi}_{23}\right)^{2}\left(\phi_{23} \phi_{3} \Psi_{\mathrm{R}}\right)^{2}\right] .
\end{aligned}
$$

Inserting the vacuum alignment and $\mathrm{VEVs}$ given in (5a)(5d), the effective Majorana mass matrix takes the form

$$
M_{\mathrm{RR}}=\left(\begin{array}{lll}
\sqrt[3]{\epsilon_{v}^{8}} & 0 & 0 \\
0 & \epsilon_{v}^{2} & \epsilon_{v}^{2} \\
0 & \epsilon_{v}^{2} & 1
\end{array}\right) \sqrt[3]{\epsilon_{v}^{8}} \frac{\left\langle\Phi_{\mathrm{R}}\right\rangle^{2}}{M}
$$

This Majorana mass matrix is diagonal up to corrections of order $\epsilon_{v}^{2}$. Furthermore, the right-handed neutrino-mass eigenstates are hierarchical $\left(\epsilon_{v}^{3}: \epsilon_{v}^{2}: 1\right)$. If we allow for a new expansion parameter $\epsilon_{v} \lesssim \epsilon_{u}^{2}$, we fulfill the requirements for "sequential dominance", and the effective PMNS matrix exhibits large mixing angles. For special choices of the order-one coefficients, specific patterns (tri-bimaximal, golden-ratio) that fit actual data are possible.

Concluding, a model with flavour-triplet Higgses and flavour-triplet flavons, supplemented by the extra symmetries listed in Table 1, is able to qualitatively explain the observed SM flavour structure.

\section{Flavon decuplet model}

As we have shown in the previous section, a model with only flavour-triplet flavons can qualitatively reproduce the correct structure of the fermion mass and mixing matrices. However, to achieve this success, we have to impose additional symmetries which eliminate terms that are formally leading, but would generate an unwanted flavour structure. The assignment of extra quantum numbers may be considered ad hoc and unnatural. Therefore, we should consider also non-fundamental flavon representations.
A generic Yukawa coupling takes the form $h \Psi_{\mathrm{L}} \Psi_{\mathrm{R}}$, in the effective theory. The prefactor of such a term, considered as a symmetry-breaking spurion, has to transform according to one of the terms in the reduction of the tensor product: $\mathbf{3} \otimes \mathbf{3} \otimes \mathbf{3}=\mathbf{1} \oplus \mathbf{8} \oplus \mathbf{8} \oplus \mathbf{1 0}$.

The simplest possibility for such a spurion is the VEV of a single flavon field, which has to transform according to either one of the singlet, octet or anti-decuplet representations of $\mathrm{SU}(3)_{\mathrm{F}}$. Further low-lying representations can occur only as a product. Therefore, the sextet representation is not very promising, since it leads to similar problems as the triplet case considered above.

The next larger representation of $\mathrm{SU}(3)_{\mathrm{F}}$ is the octet. At each order in the $1 / M$ expansion, there are possible terms that generate a Yukawa coupling once the octet VEV is inserted. However, if we explicitly construct the invariant operators, we find no operator that singles out the top Yukawa coupling. The resulting quark-mass pattern is very different from the observed one, which should have a dominant entry for the third generation. Hence, while octet-induced structures may be present in the full theory, they should be subdominant and merely act as perturbations to a Yukawa term with a different origin.

The decuplet representation of $\mathrm{SU}(3)_{\mathrm{F}}$ is more promising. It is a natural choice, since it is the symmetric product of three triplets: $\mathbf{1 0}_{i j k}=\left(\mathbf{3}_{i} \otimes \mathbf{3}_{j} \otimes \mathbf{3}_{k}\right)_{\text {sym }}$. In the following, we will investigate how far we can go by considering only decuplet flavons. Just like in the triplet case, we will keep the discussion on a semi-quantitative level and just determine hierarchy patterns that can originate from such a model. A full quantitative analysis would require a detailed scan over a rather large parameter space, which we defer to future work.

\subsection{Field content}

We study a model with the same basic symmetry (1) as in the triplet-flavon case. Matter and Higgs fields are in triplet representations. However, we put the flavon fields that are primarily responsible for flavour-symmetry breaking in the $\mathbf{1 0}$ and $\overline{\mathbf{1 0}}$ representations of $\mathrm{SU}(3)_{\mathrm{F}}$. The interactions that involve these fields generate the desired flavour structure in a straightforward and simple way.

The breaking of SU(3)F proceeds in two steps by VEVs of the decuplet flavons $\phi_{i=2,3}$. Supersymmetry requires Dterm flatness, so we also introduce their conjugate fields $\bar{\phi}_{i}$. The latter will aid in generating the light masses as subdominant contributions. The PS-breaking multiplet $\Phi_{R}$, which will be associated with neutrino-mass generation, is also in the flavour-triplet representation. The field content that is relevant for the flavour structure is shown in Table 2. Similar to the triplet model described above, we do not discuss the precise dynamics of PS and flavour-symmetry breaking, and 
Table 2 Transformation of the Higgs, matter and flavon superfields under the gauge and flavour symmetries in the flavon decuplet model

\begin{tabular}{lclll}
\hline Field & SU $(3)_{\mathrm{F}}$ & PS & $Z_{4}$ & $Z_{2}^{\mathrm{R}}$ \\
\hline$\Psi_{\mathrm{L}}$ & 3 & $(4,2,1)$ & 1 & - \\
$\Psi_{\mathrm{R}}$ & 3 & $(\overline{4}, 1,2)$ & 1 & - \\
$h$ & 3 & $(1,2,2)$ & 1 & + \\
$\phi_{3}$ & $\overline{10}$ & $(1,1,1)$ & 1 & + \\
$\phi_{2}$ & $\overline{10}$ & $(1,1,1)$ & 1 & + \\
$\bar{\phi}_{3}$ & 10 & $(1,1,1)$ & 3 & + \\
$\bar{\phi}_{2}$ & 10 & $(1,1,1)$ & 0 & + \\
$\Phi_{\mathrm{R}}$ & $\overline{3}$ & $(4,1,2)$ & 1 & + \\
\hline
\end{tabular}

omit extra fields that restore left-right symmetry and cancel gauge anomaly contributions.

The VEV structure is chosen such that $\phi_{3}$ is responsible for the third generation masses. $\phi_{2}$ gives masses to the second generation. The mass of the light quarks and the Cabibbo angle are generated by higher order corrections. The VEVs for the decuplets and anti-decuplets must coincide, so SUSY is not broken via the D-terms. For simplicity we choose the Higgs VEV to be diagonal in flavour space. In summary, the VEV structure is given by

$$
\begin{aligned}
& \left\langle\phi_{3}\right\rangle_{333}=\left\langle\bar{\phi}_{3}\right\rangle_{333} \sim \epsilon, \\
& \left\langle\phi_{2}\right\rangle_{i j k}=\left\langle\bar{\phi}_{2}\right\rangle_{i j k} \sim \epsilon^{3} \quad \text { with } i, j, k \geq 2, \\
& \langle h\rangle=(1,1,1) v_{\mathrm{MSSM}}, \\
& \left\langle\Phi_{\mathrm{R}}\right\rangle=(0,0,1) v_{\Phi} .
\end{aligned}
$$

\subsection{Yukawa potential}

Looking at the Yukawa potential, any model contains the term $Y_{0}(6)$ at dimension three which does not involve flavons at all. It can be removed by imposing a discrete symmetry. In the decuplet model, we choose a $Z_{4}$ symmetry. This will be the only extra symmetry that we need for a viable phenomenology.

The leading terms that do contribute have dimension four. They take the simple form (suppressing all flavour indices)

$W_{\text {lead }}=\frac{y_{1}}{M} \phi_{3} \Psi_{\mathrm{L}} \Psi_{\mathrm{R}} h+\frac{y_{2}}{M} \phi_{2} \Psi_{\mathrm{L}} \Psi_{\mathrm{R}} h$.

The sub-leading terms of dimension five are given by

$$
W_{\mathrm{dim} 5} \sim \frac{1}{M^{2}}\left[h \phi_{3} \bar{\phi}_{2} \Psi_{\mathrm{L}} \Psi_{\mathrm{R}}+h \phi_{2} \bar{\phi}_{2} \Psi_{\mathrm{L}} \Psi_{\mathrm{R}}\right] .
$$

The superpotential which consists of these terms generates, after flavour-symmetry breaking, a Yukawa matrix consistent with the one given in (3). As mentioned above, $\epsilon$ should differ in the up and down sector. We obtain
$Y_{u / v} \sim\left(\begin{array}{ccc}0 & \epsilon_{u}^{3} & \epsilon_{u}^{3} \\ \epsilon_{u}^{3} & \epsilon_{u}^{2} & \epsilon_{u}^{2} \\ \epsilon_{u}^{3} & \epsilon_{u}^{2} & 1\end{array}\right) \epsilon_{u}, \quad Y_{d / l} \sim\left(\begin{array}{ccc}0 & \epsilon_{d}^{3} & \epsilon_{d}^{3} \\ \epsilon_{d}^{3} & \epsilon_{d}^{2} & \epsilon_{d}^{2} \\ \epsilon_{d}^{3} & \epsilon_{d}^{2} & 1\end{array}\right) \epsilon_{d}$

For completeness, we list also the next order in the expansion, namely the dimension-six operators

$$
\begin{aligned}
W_{\text {dim6 }} \sim & \frac{1}{M^{3}} h \Psi_{\mathrm{L}} \Psi_{\mathrm{R}}\left[\bar{\phi}_{3} \bar{\phi}_{3} \bar{\phi}_{3}+\phi_{3} \phi_{3} \bar{\phi}_{3}+\phi_{2} \phi_{3} \bar{\phi}_{3}\right. \\
& \left.+\phi_{2} \phi_{2} \bar{\phi}_{3}+\phi_{3} \bar{\phi}_{2} \bar{\phi}_{2}+\phi_{2} \bar{\phi}_{2} \bar{\phi}_{2}\right] .
\end{aligned}
$$

This yields a subdominant contribution,

$Y_{\mathrm{sub}}^{f} \sim\left(\begin{array}{ccc}\epsilon^{6} & \epsilon^{8} & \epsilon^{6} \\ \epsilon^{8} & \epsilon^{6} & \epsilon^{6} \\ \epsilon^{6} & \epsilon^{6} & \epsilon^{2}\end{array}\right) \epsilon$.

With suitable order-one coefficients inserted, we obtain a phenomenologically viable flavour structure in the quark and charged lepton sector.

\subsection{Neutrino masses and Lepton mixing}

A neutrino mass and mixing structure that is consistent with neutrino data can be generated by a see-saw mechanism with sequential dominance (cf. [24] and Sect. 2). The main ingredient is a near-diagonal and hierarchical Majorana matrix for the right-handed neutrinos. In the current model, the relevant part of the superpotential contains terms of dimension four and higher,

$$
\begin{aligned}
W_{\text {Maj }} \sim & \frac{1}{M}\left(\Psi_{\mathrm{R}} \Phi_{\mathrm{R}}\right)^{2}\left[1+\frac{1}{M^{2}}\left(\phi_{3} \bar{\phi}_{3}+\phi_{2} \bar{\phi}_{3}+\bar{\phi}_{2} \bar{\phi}_{2}\right)\right. \\
& \left.+\frac{1}{M^{3}}\left(\phi_{3} \bar{\phi}_{2} \bar{\phi}_{3}+\phi_{2} \bar{\phi}_{3} \bar{\phi}_{2}\right)\right] .
\end{aligned}
$$

We obtain a Majorana mass matrix for the right-handed neutrinos which takes the form

$M_{\text {Maj }} \sim\left(\begin{array}{ccc}\epsilon_{v}^{6} & \epsilon_{v}^{7} & \epsilon_{v}^{5} \\ \epsilon_{v}^{7} & \epsilon_{v}^{4} & \epsilon_{v}^{4} \\ \epsilon_{v}^{5} & \epsilon_{v}^{4} & 1\end{array}\right) M_{N_{\mathrm{R}}}$

This matrix is nearly diagonal and exhibits a hierarchical pattern with eigenvalues of order $\epsilon_{v}^{6}: \epsilon_{v}^{4}: 1$. We need a different expansion parameter $\epsilon_{v}<\epsilon_{u}$ in the neutrino sector to fulfill the conditions for sequential dominance. By inserting order-one coefficients and allowing for CP-violating phases, we should be able to fit flavour data, even if no additional terms and fields contribute. 


\section{Conclusions}

We have studied supersymmetric models with a Pati-Salam gauge group and a SU(3) F flavour symmetry where the Higgs bi-doublet occurs in three generations and transforms as a flavour triplet. The flavour symmetry is spontaneously broken, presumably at high energy scales, and effective Yukawa couplings are generated by a sequence of non-renormalizable interactions. Associated with spontaneous flavour breaking, there are flavon fields. The low-energy phenomenology of quarks and neutrinos apparently favors flavons in the decuplet representation of $\mathrm{SU}(3)_{\mathrm{F}}$, since few terms and a simple $Z_{4}$ extra symmetry qualitatively generate the observed hierarchies in the quark and neutrino data.

Since a qualitative description of flavour data is possible, a next step, which we did not attempt in the present work, amounts to working out concrete models and fit to the complete set of low-energy flavour data. A specific signature of the scenario is the presence of extra Higgs generations with flavour quantum numbers. A further study should address the Higgs potential and any impact of Higgs-generation mixing on flavour data. However, gauge-coupling unification with intermediate left-right and Pati-Salam symmetries is consistent with the presence of multiple Higgs generations at low energies, so dedicated collider searches are definitely worthwhile.

Acknowledgments This work has been supported by a Coordinated Project Grant of the Faculty of Science and Technology, University of Siegen. We acknowledge discussions with T. Feldmann, T. Mannel, K. Schnitter, C. Luhn.

Open Access This article is distributed under the terms of the Creative Commons Attribution License which permits any use, distribution, and reproduction in any medium, provided the original author(s) and the source are credited.

Funded by $\mathrm{SCOAP}^{3}$ / License Version CC BY 4.0.

\section{References}

1. G. Aad et al. [ATLAS Collaboration] Phys. Lett. B 716, 1 (2012). arXiv:1207.7214 [hep-ex]

2. S. Chatrchyan et al. [CMS Collaboration] Phys. Lett. B 716, 30 (2012). arXiv:1207.7235 [hep-ex]

3. T.D. Lee, Phys. Rev. D 8, 1226 (1973)
4. S. Weinberg, Phys. Rev. Lett. 37, 657 (1976)

5. Y. Grossman, Nucl. Phys. B 426, 355 (1994). hep-ph/9401311

6. V. Keus, S.F. King, S. Moretti, JHEP 1401, 052 (2014). arXiv:1310.8253 [hep-ph]

7. I.P. Ivanov, E. Vdovin, Eur. Phys. J. C 73, 2309 (2013). arXiv: 1210.6553 [hep-ph]

8. A. Aranda, C. Bonilla, J.L. Diaz-Cruz, Phys. Lett. B 717, 248 (2012). arXiv:1204.5558 [hep-ph]

9. J.L. Hewett, T.G. Rizzo, Phys. Rept. 183, 193 (1989)

10. S.F. King, S. Moretti, R. Nevzorov, Phys. Lett. B 634, 278 (2006). hep-ph/0511256

11. F. Hartmann, W. Kilian, K. Schnitter, JHEP 1405, 064 (2014). arXiv:1401.7891 [hep-ph]

12. G. Altarelli, F. Feruglio, Nucl. Phys. B 741, 215 (2006). hep-ph/0512103

13. J.C. Pati, A. Salam, Phys. Rev. D 8, 1240 (1973)

14. J.C. Pati, A. Salam, Phys. Rev. D 10, 275 (1974). [Erratum-ibid. D 11 (1975) 703].

15. C. Arbelaez, R.M. Fonseca, M. Hirsch, J.C. Romao, Phys. Rev. D 87, 7, 075010 (2013). arXiv:1301.6085 [hep-ph]

16. F. Braam, J. Reuter, D. Wiesler, AIP Conf. Proc. 1200, 458 (2010). arXiv:0909.3081 [hep-ph]

17. W. Kilian, J. Reuter, Phys. Lett. B 642, 81 (2006). hep-ph/0606277

18. R. Howl, S.F. King, Phys. Lett. B 652, 331 (2007). arXiv:0705.0301 [hep-ph]

19. G. Altarelli, F. Feruglio, Rev. Mod. Phys. 82, 2701 (2010). arXiv:1002.0211 [hep-ph]

20. S.F. King, C. Luhn, Rept. Prog. Phys. 76, 056201 (2013). arXiv:1301.1340 [hep-ph]

21. S.F. King, A. Merle, S. Morisi, Y. Shimizu, M. Tanimoto, New J. Phys. 16, 045018 (2014)

22. S.F. King, Rept. Prog. Phys. 67, 107 (2004). hep-ph/0310204

23. S.F. King, G.G. Ross, Phys. Lett. B 520, 243 (2001). hep-ph/0108112

24. S.F. King, G.G. Ross, Phys. Lett. B 574, 239 (2003). hep-ph/0307190

25. I. de Medeiros, Varzielas, G.G. Ross. Nucl. Phys. B 733, 31 (2006). hep-ph/0507176

26. S.F. King, M. Malinsky, JHEP 0611, 071 (2006). hep-ph/0608021

27. S. Antusch, S.F. King, Nucl. Phys. B 705, 239 (2005). hep-ph/0402121

28. S.F. King, JHEP 0508, 105 (2005). hep-ph/0506297

29. R.G. Roberts, A. Romanino, G.G. Ross, L. Velasco-Sevilla, Nucl. Phys. B 615, 358 (2001). hep-ph/0104088

30. P. Minkowski, Phys. Lett. B 67, 421 (1977)

31. T. Yanagida, Conf. Proc. C 7902131, 95 (1979)

32. M. Gell-Mann, P. Ramond, R. Slansky, Conf. Proc. C790927, 315 (1979)

33. S.F. King, Nucl. Phys. B 562, 57 (1999). hep-ph/9904210 